\title{
Wave Equation of a Particle with the Exponential Hamiltonian in the Gravitational and Electromagnetic Field
}

\author{
N. Perković, M. Stojić
}

\begin{abstract}
This paper presents a unified view of the relativistic motion of a particle in the gravitational and electromagnetic field. Deliberationhas been carried out withinthe Dirac quantum mechanical theory by using exponential metrics. This theoretical concept brings us to an algebraic sixth-degree equation and solving the equation isa complex mathematical problem. In this paper, we show the solutionfor a basic state of the system and indicated quantum gravitational effects in the area of Planck dimensions.

Index Terms - relativistic motion, sixth-degree equation .
\end{abstract}

\section{INTRODUCTION}

Relativistic description of a body state mass $m$ in the gravitational field of a source mass $M$, where $M \gg m$, will be conducted by using Einstein's mass-energy relation $E=$ $m c^{2}[1,2]$.

A dotted body of the mass $m$ is located in the gravitational field

$\vec{g}=-\frac{R c^{2}}{|\vec{r}|^{3}} \vec{r},(1)$

where $R=\frac{G M}{c^{2}}$ is a gravitational radius.

The gravitational force between the two masses will be:

$\vec{F}=-\frac{R c^{2}}{|\vec{r}|^{3}} m \vec{r}$.

Work ofthe force $\vec{F}$ in the gravitational field $\vec{g}$ equals the change of internal energy

$$
d U=-\vec{F} \mathrm{~d} \overrightarrow{\mathrm{r}}=\frac{R c^{2} m}{|\vec{r}|^{2}} \mathrm{~d} \overrightarrow{\mathrm{r}} .(3)
$$

Since

$d E_{k}=-\mathrm{dU}=c^{2} d m$, (4)

a differential equation which shows the change of the body mass in the gravitational field derives from the equations (3) and (4):

$$
\frac{d m}{m}=-\frac{R}{|\vec{r}|^{2}} d|\vec{r}| \text {. }
$$

Furthermore, by integrating the equation (5) the following mass equationis obtained:

$$
m=m_{\infty} e^{R / r}(6)
$$

where $m=m_{\infty}$ when $|\vec{r}| \rightarrow \infty[3]$.

Including the mass expression from the equation (6) into theequation (3), the change of the internal energy will be:

$$
d U=\frac{R c^{2} m_{\infty} e^{R / r}}{|\vec{r}|^{2}} d \vec{r} .(7)
$$

\section{Since}

$d H=d E_{k}+d U,(8)$

from the equations (4) and (7), we get Hamiltonian particles of the mass $m$ in the gravitational field of the source $M[5]$.

$$
H=m c^{2} \mathrm{e}^{-\mathrm{R} / \mathrm{r}}(9)
$$

Hamiltonian (9) has an exponential member which shows that total energy of the particle depends on its position in the gravitational field.

\section{INTERACTION OF THE PARTICLE WITH THE} GRAVITATIONAL AND ELECTROMAGNETIC FIELD WITHIN THE DIRAC QUANTUM MECHANICAL THEORY

Using relativistic relations between energy and impulses, Hamiltonian particles can be written in the following form:

$H=e^{-R / r} c \sqrt{g^{i j} p_{i} p_{j}+m^{2} c^{2}}, i, j=1,2,3(10)$

where $g^{i j}$ isa metric tensor which, in this case, has diagonal components:

$$
\begin{aligned}
g^{11} & =e^{2 R / r}, \\
g^{22} & =r^{2} e^{2 R / r}, \quad\left(\theta=\frac{\pi}{2}\right) \\
g^{33} & =r^{2} e^{2 R / r}, \\
g^{00} & =-e^{-2 R / r} .(11)
\end{aligned}
$$

In Dirac's approach, Hamiltonian has a linear dependence on the impulse so the equation can be written:

$$
H=e^{-R / r}\left(c \vec{\alpha} \vec{p}+\beta m c^{2}\right),(12)
$$

where $\alpha$ and $\beta$ are matrices while $\vec{p}$ is the impulseof the particle mass $m$. Comparing the equations (10) and (12), the relationship between the matrices $\alpha$ and $\beta$ is obtained as well as their connection to the impulse and the metric tensor.

The following relations are established by conducting the calculation:

$$
\begin{aligned}
& \left\{\alpha_{i}, \alpha_{j}\right\}=2 g^{i j} \delta_{i j}, \delta_{i j}-\text { Kronecker symbol } \\
& \left\{\alpha_{i}, \beta\right\}=0 \\
& \alpha_{i}^{2}=\beta^{2}=1 \\
& {\left[p_{i}, \alpha^{j}\right]=0} \\
& \alpha^{i}(r)=\sqrt{g^{i i}} \alpha_{D}^{i},(13)
\end{aligned}
$$

where $\alpha_{D}^{i}$ represents Dirac's matrices.Hamiltonian operating (12) on the wavefunction $\psi$ is shown with the equation:

$$
\begin{aligned}
& H \psi=e^{-R / r} c(\vec{\alpha} \vec{p}+\beta m c) \psi=E \psi \text { (14) } \\
& \text { or: } \\
& \vec{\alpha} \cdot \vec{p} \psi=\left(\frac{E}{c} e^{R / r}-\beta m c\right) \psi \cdot(15) \\
& \text { By applying the relations (13), the impulse } p \text { can be } \\
& \text { replaced by a covariant differential operator } D_{k}
\end{aligned}
$$


$p_{k}=-i \hbar D_{k}=-i \hbar\left(\partial_{k}+\Gamma_{k v}^{\square}\right), \quad$ 目, $v=0,1,2,3(16)$

where $\Gamma_{k v}^{\square}$ represents Christoffel symbols. Taking the wave function $\psi$ in the following form

$\psi=\left(\begin{array}{l}\psi_{1} \\ \psi_{2}\end{array}\right)=\frac{1}{r}\left(\begin{array}{cc}F & Y_{+} \\ i & G Y_{-}\end{array}\right)(17)$

and using already known properties of $\alpha$ matrix when operating on the wave function, i.e.

$$
\begin{array}{r}
\beta(\hbar+\vec{\sigma} \cdot \vec{L}) \psi=-(I+1 / 2) \psi \\
\frac{\vec{\alpha} \cdot \vec{r}}{|\vec{r}|} Y_{-}^{+}=-\sqrt{g^{11}} Y_{+}^{-}, \frac{|\vec{r}|^{2}}{r^{2}}=\sqrt{g_{11}}(18)
\end{array}
$$

the equation (15) takes the form:

$\hbar\left[\frac{\partial F(r)}{\partial r}+\Gamma_{11}^{1} F(r)+e^{-\frac{3}{2} R / r} \frac{I+1 / 2}{r} F(r)\right]=\left(\frac{E}{c} e^{3 R / r}+\right.$ $\left.e^{2 R / r} m c\right) G(r)$,

$\hbar\left[-\frac{\partial G(r)}{\partial r}-\Gamma_{12}^{2} G(r)+e^{-\frac{3}{2} R / r} \frac{I+1 / 2}{r} G(r)\right]=$ $\left(\frac{E}{c} e^{3 R / r}-e^{2 R / r} m c\right) F(r) .(19)$

Equations (19) that we had obtained are valid for the gravitational field of the mass source $M[4]$. The equations can also be extended to the electromagnetic field which is achieved by adding vector potential of the electromagnetic field $A_{\square}=\left(A_{i}, A_{0}\right)$ into the equation (16) whereby

$$
p_{\text {回 }} \rightarrow-i \hbar D_{\text {回 }} \rightarrow-i \hbar\left(\partial_{\text {回 }}+\Gamma_{\text {回 }}+i e A_{\text {回 }}\right) \text {. }
$$

The operation of the covariational operator $D_{\text {曰 }}$ is defined by the following equation:

$$
D_{k} \psi^{\square}=\left(\partial_{k} \psi^{\square}+\Gamma_{k v}^{\square} \psi^{v}\right) \text {. }
$$

For a simpler consideration, we will assume that magnetic components of vector potential equal zero, i.e. $A_{i}=0, i \neq$ 0 , while electric component $A_{0}=\varphi$, so ultimately the equation (16) becomes:

$$
p_{\mu}=-i \hbar D_{\mu}=-i \hbar\left(\partial_{\mu}+\Gamma_{\mu}+i e A_{\mu} \cdot \delta_{0 \mu}\right), \quad \text { ?, } v=
$$

$0,1,2,3$.

In this way the equations (19) become:

$\hbar\left[\frac{\partial F(r)}{\partial r}+\Gamma_{11}^{1} F(r)+e^{-\frac{3}{2} R / r} \frac{I+1 / 2}{r} F(r)\right]=\left(\frac{E-e \varphi}{c} e^{3 R / r}+\right.$ $\left.e^{2 R / r} m c\right) G(r)$,

$$
\hbar\left[-\frac{\partial G(r)}{\partial r}-\Gamma_{12}^{2} G(r)+e^{-\frac{3}{2} R / r} \frac{I+1 / 2}{r} G(r)\right]=
$$
$\left(\frac{E-e \varphi}{c} e^{3 R / r}-e^{2 R / r} m c\right) F(r)$. (20)

We look for the solutions of the equations (20) for a potential $\varphi=-\frac{Z e}{r}$, whereby introducing a new variable $\rho=\varepsilon r$.

By developingthe order of exponential members to the second member and transitioning to the natural system of units $(\hbar=c=1)$, the equations (20) can be written:

$$
\varepsilon\left[\frac{\partial F(r)}{\partial \rho}+\frac{I+1 / 2}{\rho} F(r)\right]=\left[E+m+\frac{R \varepsilon}{\rho}(3 E+2 m+\right.
$$

$\left.\left.\frac{Z e^{2}}{R}\right)\right] G(\rho)$,

$\varepsilon\left[-\frac{\partial G(r)}{\partial \rho}+\frac{I-1 / 2}{\rho} G(r)\right]=\left[E-m+\frac{R \varepsilon}{\rho}(3 E-2 m+\right.$ $\left.\left.\frac{Z e^{2}}{R}\right)\right] F(\rho) .(21)$

In order to make the solutions of the equations (21) regular when $r=0$ and $r=\infty$, the functions $F(\rho)$ and $G(\rho)$ are taken in the form:

$$
\begin{aligned}
& F(\rho)=\rho^{s} e^{-\rho} a_{n} \rho^{n}, \\
& G(\rho)=\rho^{s} e^{-\rho} b_{n} \rho^{n} .(22)
\end{aligned}
$$

Development coefficients of the functions $F(\rho)$ and $G(\rho)$ satisfy the following conditions:

$$
a_{n^{\prime}} \neq 0 \text {, }
$$

$b_{n^{\prime}} \neq 0$,

$a_{n^{\prime}+k}=b_{n^{\prime}+k}=0, \forall \mathrm{k} \in \mathrm{N} .(23)$

Using the functions (22) and properties of the coefficients (23), the equations (21) become:

$$
\begin{aligned}
& \quad \varepsilon(s+n+1+I+1 / 2) a_{n+1}-\varepsilon a_{n}=(E+m) b_{n}+ \\
& \varepsilon R\left(3 E+2 m+\frac{Z e^{2}}{R}\right) b_{n+1}, \\
& \quad \varepsilon(-s-n-1+I-1 / 2) b_{n+1}+\varepsilon b_{n}=(E-m) a_{n}+ \\
& \varepsilon R\left(3 E-2 m+\frac{Z e^{2}}{R}\right) a_{n+1} .(24)
\end{aligned}
$$

Comparing the two equations (24) for the selected $n$, we obtain a relationfor determiningeigenvalues. For instance, there is a relation for $n=n^{\prime}$ :

$\varepsilon^{2}=m^{2}-E^{2}$.(25)

For $n=-1$ we obtain the following equation:

$$
(s+1 / 2)^{2}=I^{2}-R^{2}\left(3 E+\frac{Z e^{2}}{R}\right)^{2}+4 m^{2} R^{2} \cdot(26)
$$

And forn $=n^{\prime}-1$ we obtain the following equation:

$\varepsilon\left(s+n^{\prime}+1 / 2\right)-R\left(3 E^{2}-2 m^{2}\right)-Z e^{2} E=0 .(27)$

From the equations (26) and (27) we can eliminate the parameter $s$, andby using the relation (25), the eigenvalue equationis obtained in the following form:

$6 \varepsilon^{3} R n^{\prime}+\varepsilon^{2}\left(n^{\prime 2}-I^{2}-R^{2} m^{2}\right)-2 \varepsilon n^{\prime} R m^{2}+$

$m^{2}\left(R^{2} m^{2}+Z^{2} e^{4}\right)=2 Z e^{2} \sqrt{m^{2}-\varepsilon^{2}}\left(n^{\prime} \varepsilon-R m^{2}\right)$

By solving the equation (28), the energy spectrum is obtained.Since it is a sixth-degreeequation, we will look for the solutions of $n^{\prime}=0$. Thebasic state energy for the gravitational and electric field is:

$$
E_{1,2,3,4}=\frac{ \pm R m^{2} Z e^{2} \pm I m \sqrt{I^{2}+R^{2} m^{2}-Z^{2} e^{4}}}{I^{2}+R^{2} m^{2}} \text {.(29) }
$$

$s>0$ is required for the regularity of the equations (21), which is satisfied when:

$$
m^{2}>\frac{1}{4}\left(3 E+\frac{Z e^{2}}{R}\right)^{2} \text {. }
$$

Eigenvalues(28) $\quad \varepsilon_{n}=\sqrt{m^{2}-E_{n}{ }^{2}}$ are givenby the sixth-degree equation, so solving the equation for $n^{\prime}>0$ is not as simple, because with one solution thatmatches the matter-antimatter, it exists five more solutions that need to be defined.

\section{CONCLUSION}

This paper shows a relativistic description ofthe motion of the particle in the gravitational and electromagnetic field. The calculation has been performed withinthe Dirac quantum mechanical theoryby using exponential metrics. The gravitational and electromagnetic field are connected through the covariant operator $D_{\text {回 }}$.Moreover, the algebraic sixth-degree eigenvalue equation is obtained by solving the system of differential equations. Since solving the algebraic sixth-degree equation presents a complex mathematical problem, an analysis of the solutions to the basic state has been performed while other states are to be explored. Equations that are obtained also describe gravitational quantum effects which occur when the gravitational radius $R$ 
is in the area of Planck wavelengths, i.e. $R \approx \lambda_{p}$.

\section{REFERENCES}

[1] L. D. Landau and E. M. Lifshitz, The Classical Theory of Fields, Pergamon Press, 1971.

[2] A. Einstein, The Meaning of Relativity, Princeton University Press, 2005.

[3] N. Perković, M.Stojić, Relativistic Equations of Motion Based on Equality of Heavy and Inertial Mass, International Journal of New Technology and Research (IJNTR) ISSN:2454-4116, Volume-4, Issue-11, November 2018 Pages 44-47

[4] M. Martinis, N.Perković, On the Eigenvalue Problem of the Dirac Hamiltonian in a Singular Spacetime Background, International Journal of New Technology and Research (IJNTR) ISSN:2454-4116, Volume-4, Issue-2, February 2018 Pages 100-102 100

[5] M. Martinis, N. Perković, Relativistic Modification of Newtonian Gravity Law, International Journal of New Technology and Research (IJNTR) ISSN:2454-4116, Volume-4, Issue-2, February 2018 Pages $42-46$ 